\title{
DC-SC PHOTOINJECTOR WITH LOW EMITTANCE AT PEKING UNIVERISTY
}

\author{
R. Xiang, Y. Ding, J. Hao, S. Huang, X. Lu, S. Quan, B. Zhang, K. Zhao* \\ SRF Laboratory, IHIP, Peking University, Beijing 100871, China
}

\begin{abstract}
High average power Free Electron Lasers require the high average current electron beams with the low emittance. The DC-SC photoinjector developed at Peking University, directly combining a DC Pierce gun with an SRF cavity, can produce the high brightness beam with moderate bunch charge and high duty factor. In order to optimize the transverse emittance which sharply increases at the beginning of the DC gun, a carefully design for the DC gap and the cavity shape is needed. In this paper, the simulation of an upgraded design has been performed. This photoinjector, consisting of a DC gap and a $2+1 / 2$ cell SRF cavity, can produce $4.2 \mathrm{MeV}$ electron beams with $100 \mathrm{pC}$ per bunch and $1.1 \mu \mathrm{m}$ normalized emittance.
\end{abstract}

\section{INTRODUCTION}

The conventional RF photoinjectors can provide the electron pulses with high charge and low transverse emittance, but it is little possible to use them as the electron sources for the high average power FELs and ERL. Because the superconducting cavity can work in the $\mathrm{CW}$ mode, the photoinjectors based on superconducting cavity make the best choice to produce the high average current electron beams. At Peking University, DC-SC photoinjector is developed to provide the electron source for PKU-FEL, which is designed for high average power infrared $(5 \sim 10 \mu \mathrm{m})$ and $\mathrm{THz}(100 \sim 3000 \mu \mathrm{m})$ light source [1]. This DC-SC photoinjector is a new design different from Rossendorf [2], BNL [3] and Jefferson lab [4]. By integrating a small DC Pierce gun with the superconducting cavity, it is free of the compatibility of the superconducting cavity and the normal conducting photocathode.

\section{1+1/2 CELL INJECTOR TEST}

Since January 2003, the DC-SC photoinjector based on the $1+1 / 2$ cell niobium cavity has been constructed [5]. Core elements of this gun are the SC cavity, the DC Pierce gun, the photocathode, the drive laser, the diagnose and the RF system. The $\mathrm{Cs}_{2} \mathrm{Te}$ photocathode is placed in the centre of the Pierce cathode. Starting from the cathode, the electrons pass through the centre hole of the anode and enter into the $1+1 / 2$ cell cavity. The photolayer is deposited on the tip of a stainless steel stem in a special preparation chamber connected to the DC gun. With this design, the cathode can be transferred into the injector by a manipulator without any vacuum break or cavity warm up.

The 3D-view of the test facility is presented in figure 1,

\footnotetext{
* Supported in part by Chinese Ministry of Science and Technology under the National basic Research Projects (No.2002CB713602) \#k.zhao@pku.edu.cn
}

including the cavity, the cryostat, the DC gap and the RF main coupler. After filling the Liquid Helium vessel by a Dewar, the gun was operated at $4.2 \mathrm{~K}$ with the quality factor of $2 \times 10^{8}$ and the average gradient reached $6 \mathrm{MV} / \mathrm{m}$. The first beam loading has been successfully performed in 2004. Up to now, the maximum energy gain of $1 \mathrm{MeV}$ was achieved at $4.2 \mathrm{~K}$. When the beam energy was 500 $\mathrm{keV}$ and the average current was $270 \mu \mathrm{A}$, the transverse emittance has been measured about $5 \mu \mathrm{m}$, and the energy spread about $35 \mathrm{keV}$ [6]. Now the beam loading experiment at $2 \mathrm{~K}$ is on the schedule to improve accelerating gradient of superconducting cavity and thus to attain higher energy. On the other hand, the test on the $1+1 / 2$ cell gun indicates that an upgrade is needed to fulfil the requirements of PKU-FEL $[1,7,8]$.

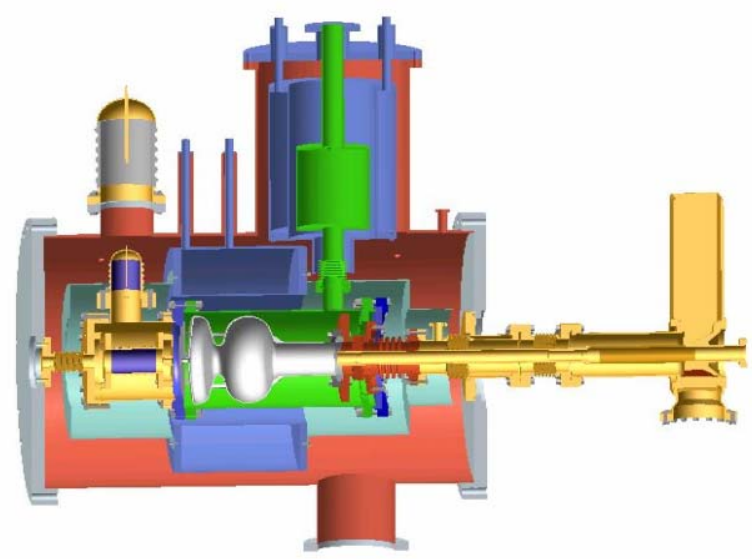

Figure 1: 3D-view of the DC-SC injector test facility: DC gun, $1+1 / 2$ cell $\mathrm{Nb}$ cavity, cryostat, and RF power coupler

\section{SIMULATION OF NEW INJECTOR}

In parallel to the prototype test of $1+1 / 2$ cell gun, the simulation of the $2+1 / 2$ cell photoinjector has been done. The goals of the new design is to produce the beams with the energy around $4 \mathrm{MeV}$, the bunch charge of $60 \sim 100 \mathrm{pC}$ and the transverse emittance less than $2 \mu \mathrm{m}$.

The device begins with the $1+1 / 2$ cell DC-SC injector prototype. $2+1 / 2$ cell cavity has been chosen because by assuming that the average accelerating field of the SC cavity is $15 \mathrm{MV} / \mathrm{m}$, a $2+1 / 2$ cell cavity can accelerate the electrons to above $4 \mathrm{MeV}$. The geometry of the 2 full cells is based on the TESLA design [9], and the half cell is optimized to a 0.6 cell cavity with a slope wall of $5 \mathrm{deg}$. Figure 2 gives out the field pattern and the near-axis field in the cavity simulated by the code SUPERFISH. The RF field near the hole of the half cavity has the focusing force on the electrons, which is useful in the control of emittance, and moreover, this function is rising via the radial distance. 
In order to keep the DC part as compact as possible, the voltage on the cathode is still $-70 \mathrm{kV}$, which means the effort on the design or purchase of the new high voltage elements could be saved. The slope of the poles in the DC gun is $16 \mathrm{deg}$, and the distance between the poles is $9 \mathrm{~mm}$. There exists a hole in the anode with the diameter of 8 $\mathrm{mm}$ through which the electrons enter the half cell cavity. To prevent the RF power loss caused by this hole, the distance between the anode of the Pierce gun and the wall of the half cell is designed to $13 \mathrm{~mm}$. The maximum field along the surface of $\mathrm{DC}$ gun is $12 \mathrm{MV} / \mathrm{m}$, which is far below the threshold of field emittance.

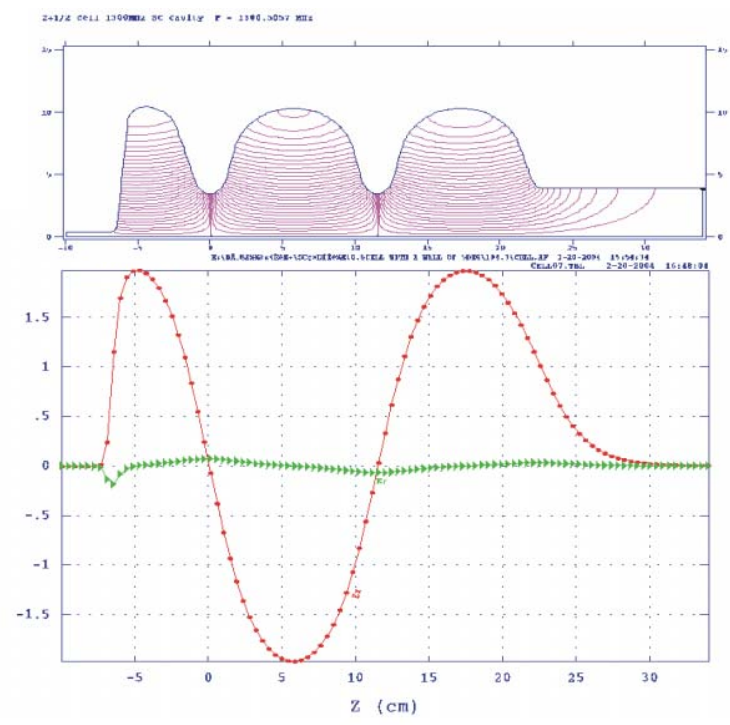

Figure 2: Field pattern of $2+1 / 2$ cavity and near-axis electric field $(\mathrm{r}=2 \mathrm{~mm})$.

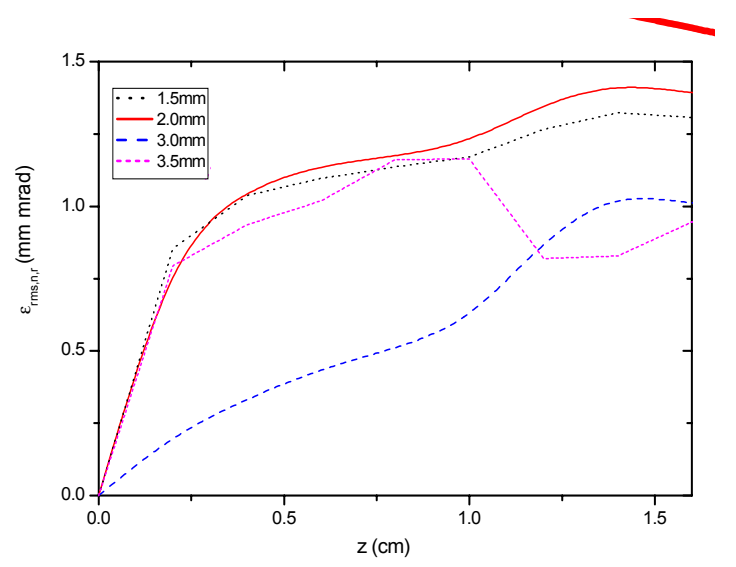

Figure 3: Transverse emittance of the beam with different radius in $\mathrm{DC}$ gun.

The $\mathrm{Cs}_{2} \mathrm{Te}$ photocathode is driven by the $266 \mathrm{~nm}$ ultraviolet laser, which is reformed to radial uniform and lognitudinal Gauss distribution. As known already, the smaller charge density benefits the smaller transverse emittance, and the lognitudinal Gauss distribution can concentrate the electrons in the small RF phase region and therefore results in a small energy spread. Figure 3 presents the transverse emittance in DC gun. A larger radius will lead to the less space charge force and consequently decrease the emittance. But limited by the size of the hole ( $8 \mathrm{~mm}$ diameter), too large beam will loose electrons on anode. The radius of laser spot is optimized to $3 \mathrm{~mm}$ to prevent too strong space charge force and also to reduce the effect of thermal emittance caused by the photoelectrons themselves. The crucial problem is whether the emittance increment in the DC gap is beyond our tolerance. The transverse rms emittance in the end of the DC gun is as large as the expected one for the whole injector, but in the following content we can find the rms emittance is still under control.

For the design of DC-SC photoinjector, the main open question was the relative large transverse emittance. In order to lower that, the emittance compensation theory is applied in the new injector [10], which is realized by adding a $20 \mathrm{~cm}$ solenoid as the compensation in the beam line. The position is $54 \mathrm{~cm}$ forward the SC cavity and the static magnetic field is chosen as 835 Gauss (see figure 4). The beam dynamics simulation has been done with Parmela code [11], and in the simulation 20000 reference particles are used. Table 1 presents the main parameters of the injector, including the beam quality in the position where the smallest emittance appears as the position " $\mathrm{B}$ " in the figure 4 . The transverse normalized emittance is as small as $1.1 \mu \mathrm{m}$, and the energy spread is $0.60 \%$. At the same time, the bunch shrinks in the radius and length.

Table 1: The parameters of the new injector

\begin{tabular}{lll}
\hline Cavity & $\mathrm{E}_{\text {acc }}$ & $15 \mathrm{MV} / \mathrm{m}$ \\
& $\mathrm{B}_{\max } / \mathrm{E}_{\max }$ & $2.4561 \mathrm{mT} /(\mathrm{MV} / \mathrm{m})$ \\
& $\mathrm{E}_{\max } / \mathrm{E}_{0}$ & 2.1841 \\
& $\mathrm{Q}_{0}$ & $8.62 \times 10^{9}$ \\
\hline Laser & $\varphi_{\text {simul. }}$ & $225^{\circ}$ \\
& $\mathrm{r}_{\text {edge }}$ & uniform, $3 \mathrm{~mm}$ \\
& $\mathrm{~L}_{\mathrm{FWHM}}$ & Gauss, $8 \mathrm{ps}$ \\
\hline Bunch & Charge & $100 \mathrm{pC}$ \\
& $\mathrm{E}$ & $4.225 \mathrm{MeV}$ \\
& $\Delta \mathrm{E} / \mathrm{E}$ & $0.60 \%$ \\
& $\varepsilon_{\text {rms,n, } \mathrm{x}}$ & $1.134 \mathrm{~mm} \cdot \mathrm{mrad}$ \\
& $\varepsilon_{\text {rms,n, } \mathrm{z}}$ & $9.980 \mathrm{deg}-\mathrm{keV}$ \\
& $\mathrm{r}_{\text {edge }}$ & $2.5 \mathrm{~mm}$ \\
& $\mathrm{~L}_{\text {FWHM }}$ & $5.6 \mathrm{ps}$ \\
\hline
\end{tabular}

On the other hand, from figure 4, this place for the smallest emittance is $268 \mathrm{~cm}$ away from the cathode (position "B"), before which a beam waist exists in the position of $230 \mathrm{~cm}$ (position "A"). In fact, this waist 
position could be the best place for the next accelerating element, where simultaneously $143 \mathrm{~cm}$ forward from the solenoid. This is suitable for the cryomodule, the beam diagnose, the laser input port and the other engineering arrangement between the injector and the main linac.

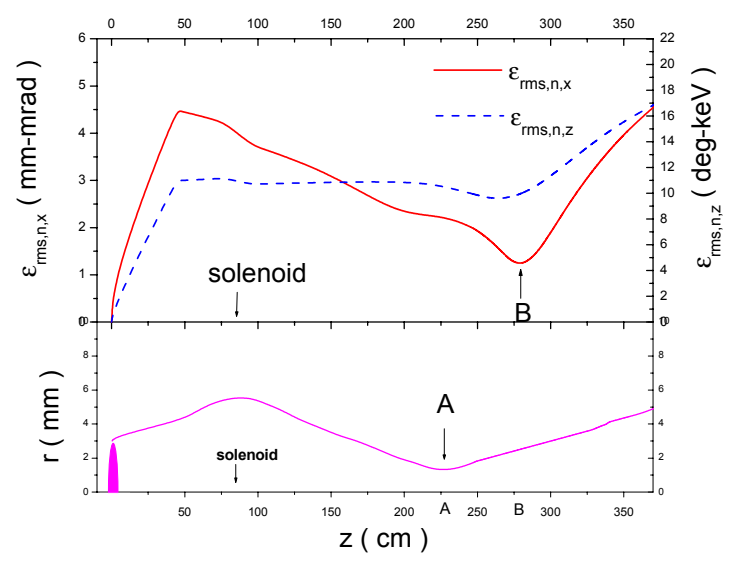

Figure 4: the transverse emittance and the bunch radius along the $\mathrm{z}$ axis.

One of the advantages of DC-SC injector is that the cathode works in the low temperature and thus the thermal emittance will be moderately reduced. According to ref. 12 , the thermal emittance $\varepsilon_{\text {th }}$ of $\mathrm{Cs}_{2} \mathrm{Te}$ can be estimated by

$$
\varepsilon_{t h}=\frac{R}{2 \sqrt{3}} \sqrt{\frac{2 E k i n}{m_{0} c^{2}}}=1.247 \mu m
$$

Here $\mathrm{R}$ is the radius of the laser spot on the cathode and $E_{\text {kin }}$ is the kinetic energy of the electrons emitted out from the photocathode. Among these variables, kinetic energy of the electrons depends on the working temperature of cathode, the energy of driver laser photos, and the band gap of $\mathrm{Cs}_{2} \mathrm{Te}$ semiconductor. In DC-SC injector, the cathode is placed in $77 \mathrm{~K}$ surrounding, so the thermal emittance of the $\mathrm{Cs}_{2} \mathrm{Te}$ can be calculated as $1.247 \mu \mathrm{m}$, which is the original emittance for the electron beam. When the thermal emittance is integrated with the beam kinetic emittance $\varepsilon_{\mathrm{rms}, \mathrm{n}}$ after the DC and RF field, the total beam emittance of DC-SC injector becomes $1.68 \mu \mathrm{m}$. The thermal emittance of the cold cathode should be measured by the experiments, which could be important for the practical use of the gun in the future.

\section{SUMMARY}

DC-SC photoinjector has been developed and tested for the high average power PKU-FEL project. The $4.2 \mathrm{~K}$ experiments on the prototype with $1+1 / 2$ cell cavity have proved the reliability of integration: DC gun with normal conducting photocathode and SC cavity. To provide the beam with $4 \mathrm{MeV}$ energy and low rms emittance, an upgrade version with $2+1 / 2$ cell cavity is designed, in which emittance compensation technique is adopted to get low transverse rms emittance of $1.1 \mu \mathrm{m}$. Cold photocathode has relatively low thermal emittance, and the $\mathrm{Cs}_{2} \mathrm{Te}$ photocathode working in DC-SC injector is estimated in this paper.

\section{REFERENCES}

[1] S. Huang, et al., FEL'04, Trieste, Italy, Aug 29- Sep 3, 2004.

[2] D. Janssen, et al., NIM A507 (2002)314.

[3] T. Srinivasan-Rao, et al., PAC'03, Portland, USA, May 12-16, 2003.

[4] H. Bluem, et al., EPAC'04, Lucern, Swiss, July 5-9, 2004.

[5] R. Xiang, et al., NIM A528 ( 2004) 321.

[6] X. Lu, et al., ERL'05, Newport News, VA, USA, March 19 - 23, 2005.

[7] K. Zhao, et al, NIM A483(2002)125.

[8] Y. Ding, et al., NIM A528(2004) 416.

[9] B. Aune, et al., Phys. Rev. Special Topics, Vol. 3 (2000) 092001.

[10] B.E. Carlsten, NIM A285 (1989) 313.

[11] L. Young, LA-UR-96-1835, LANL, 1996.

[12] K. Floettmann, TESLA-FEL-97-06 